\title{
A multivariate quality loss function approach for parametric optimization of non-traditional ma- chining processes
}

\author{
Shankar Chakraborty ${ }^{a^{*}}$ and Partha Protim Das ${ }^{\mathrm{b}}$
}

${ }^{a}$ Department of Production Engineering, Jadavpur University, Kolkata, West Bengal, India ${ }^{b}$ Department of Mechanical Engineering, Sikkim Manipal Institute of Technology, Sikkim, India

\begin{tabular}{l}
\hline C H R O N I C L E \\
\hline Article history: \\
Received: March 23, 2018 \\
Received in revised format: March \\
23, 2018 \\
Accepted: June 5, 2018 \\
Available online: \\
June 5, 2018 \\
\hline Keywords: \\
Multivariate loss function \\
Quality \\
Non-traditional machining \\
process \\
Process parameter \\
Response \\
Optimization \\
\hline
\end{tabular}

Due to various added advantages over the conventional material removal processes, non-traditional machining (NTM) processes have now been widely applied in different manufacturing industries. To achieve the desired response values, it is always recommended to operate these NTM processes at their optimal parametric settings. Various single response optimization techniques are already available to determine the optimal combinations of NTM process parameters for achieving maximum or minimum value of a single response. In this paper, a multivariate quality loss function approach is adopted for simultaneous optimization of responses for three NTM processes. It is observed that this approach outperforms the other multi-response optimization techniques, like desirability function, distance function and mean squared error methods with respect to the achieved response values. With modification of the corresponding objective function and constraints of the developed non-linear programming problem, it can be effectively applied to any non-traditional as well as conventional machining process as a multi-objective optimization tool.

C) 2018 by the authors; licensee Growing Science, Canada

\section{Introduction}

In other to meet the requirements of high dimensional accuracy and low surface roughness of the present day's manufacturing industries, the conventional machining methods are continuously being substituted by the non-traditional machining (NTM) processes due to their ability to achieve more consistent workpiece quality, higher production efficiency in processing of hard and tough materials, and capability of generating complex shapes. These NTM processes usually deploy mechanical, electrical, chemical or thermal energy or their combinations to remove material from the workpiece in the form of atoms/molecules enabling attainment of high degree of surface finish (Pandey \& Shan, 1980). Like the conventional machining processes, they do not use sharp cutting tools and the material removal rate is also not constrained by the mechanical properties of the work materials. These NTM processes are quite suitable to generate complex shape geometries on different difficult-to-machine materials, like ceramic-based tool materials, fibre reinforced materials, tungsten carbides, stainless steels, high speed steels, titanium-based alloys etc. Complex shape features with internal and external profiles, small holes having micro- and 
nano-dimensions, and components requiring high tolerance and surface finish can be precisely and economically machined using these processes. It is a well understood fact that the NTM processes can probably never replace the conventional machining processes currently employed in various manufacturing industries. However, these processes have ensured a steadily increasingly important role because of their improved machining capabilities and characteristics.

To exploit the fullest potential and benefits from these NTM processes for generating complex and intricate shape geometries having the required dimensional accuracy, tolerance and surface finish on various difficult-to-machine work materials, they are always required to operate at their optimal parametric settings. Identification of the most appropriate parametric mix for a specific NTM process to generate the desired shape feature on a given work material is often found to be a challenging task. For this purpose, the manufacturers' operating manuals are frequently consulted or the machining experts' opinions are regularly sorted. The existence of several conflicting machining responses, like maximization of material removal rate (MRR) and minimization of surface roughness (SR), maximization of machining efficiency and minimization of power requirement etc., also makes the identification of the optimal combination of various NTM process parameters a more difficult task. Thus, it is always recommended to deploy some mathematical tools and techniques that would provide optimal or near optimal settings of the NTM process parameters so as to meet the target response values.

\section{Literature review}

Antony (2001) applied Taguchi quality loss function approach for simultaneous optimization of multiple quality characteristics in manufacturing processes. While applying Taguchi approach and utility concept, Singh and Kumar (2006) predicted the optimal setting of turning process parameters for having the desired quality characteristics of EN24 steel turned components. Walia et al. (2006) integrated utility theory and Taguchi quality loss function for optimization of three responses, i.e. MRR, percentage improvement of surface finish and scatter of SR in a centrifugal force-assisted abrasive flow machining process. Datta and Mahapatra (2010) combined utility theory with principal component analysis and Taguchi robust design for simultaneous optimization of multiple correlated surface quality characteristics of mild steel in straight turning operation. Dave et al. (2012) proposed the application Taguchi loss function as a multi-response optimization tool for electro discharge machining (EDM) operation of Inconel 718 alloy. Gaitonde and Karnik (2012) employed Taguchi quality loss function approach to identify the best combination of cutting speed, feed, point angle and lip clearance angle for specified drill diameters to simultaneously minimize burr height and burr thickness during drilling of AISI 316L stainless steel work materials. Kumar and Kumari (2012) applied fuzzy logic and Taguchi quality loss function for modelling and simultaneous optimization of multiple performance characteristics, such as MRR and SR of polycrystalline diamond in an ultrasonic machining (USM) process. Periyanan et al. (2012) applied Taguchi loss function approach to study the influences of spindle speed, feed rate and depth of cut on MRR and SR in a micro-end milling process. Singh et al. (2012) adopted Taguchi quality loss function for identifying the optimal operating levels of wheel speed, current, pulse-on time and duty factor in an electro-discharge diamond grinding process. Three quality characteristics, i.e. MRR, wheel wear rate and average SR were subsequently optimized. Applying Taguchi quality loss function approach, Sahoo and Mohanty (2013) determined the optimal settings of cutting speed, feed and depth of cut so as to minimize cutting force and chip reduction coefficient in an orthogonal turning process. Bhuyan and Yadava (2014) considered applied voltage, electrolyte concentration, wire feed velocity and workpiece thickness as the input parameters, and SR and MRR as the responses in a travelling wire electro-chemical spark machining process. Taguchi quality loss function was then employed to determine the optimal levels of those process parameters. Das Mohapatra and Sahoo (2014) analyzed the effects of four input parameters of a wire electro discharge machining (WEDM) process, i.e. pulse-on time, pulse-off time, wire feed rate and wire tension on the geometry of machined spur gears (i.e. MRR and single pitch error). Those WEDM process parameters were subsequently optimized employing Taguchi loss function approach. Dhobe et 
al. (2014) performed parametric analysis and single objective optimization based on Taguchi methodology during electrochemical machining (ECM) of pure titanium material. Using quality loss function, multi-objective optimization of two responses, i.e. MRR and SR was also conducted. It was observed that electrolyte flow rate, applied voltage, electrolyte concentration and initial inter-electrode gap were the main process parameters affecting the considered responses. Hansda and Banerjee (2014) considered material thickness, cutting velocity and feed rate in drilling of glass fibre reinforced polyester composites, and employed Taguchi quality loss function as a multi-objective optimization tool. Koranne et al. (2014) investigated the performance of multi-layer coated tool in machining of hardened steel under high speed turning and optimized the process using quality loss function approach. Kumar (2014) studied the influences of various parameters of an USM process, i.e. type of tool material, abrasive type, slurry grit size and power rating on surface quality and micro-hardness of the machined components. Taguchi's robust design approach was effectively applied for optimizing the said process. While considering air pressure and stand-off distance as the control parameters, Padhy et al. (2014) simultaneously optimized overcut and MRR of glass materials in an abrasive jet machining process using quality loss function. Periyanan and Natarajan (2014) applied Taguchi quality loss function approach to investigate the effects of three micro-WEDM process parameters, i.e. feed rate, capacitance and voltage on MRR and SR. Rahul et al. (2017) determined the optimal settings for gap voltage, peak discharge current, pulse-on time, duty factor and flushing pressure while attaining the desired values of MRR, electrode wear rate, SR, surface crack density, white layer thickness and micro-hardness during EDM operation of Inconel 718 alloy. The derived results were also compared with those observed using principal component analysis and quality loss approach.

From the above-cited literature survey, it is clearly noticed that since few decades, parametric optimization of different NTM as well as conventional machining processes has been a topic of immense interest among the researchers. The concept of Taguchi loss function has been popularly employed for identifying the optimal parametric mixes for several machining processes to fulfil the end requirements. The Taguchi loss function is an effective tool for single-objective optimization of NTM processes. As almost all the NTM processes have multiple quality characteristics or responses, the application of Taguchi loss function miserably fails to simultaneously optimize all the considered responses. To overcome this drawback of Taguchi loss function, in this paper, a multivariate quality loss function approach is adopted which can concurrently optimize all the responses of the NTM processes under consideration.

\section{Multivariate quality loss function}

The concept of quality loss function was introduced by Genichi Taguchi in the early 1950's, which was later implemented by the major American manufacturers. In the Western Europe, it was also executed among the automotive and aircraft industries. The classical definition of quality control is based on lower and upper specification limits (LSL and USL) as boundaries between the acceptable and unacceptable performance.

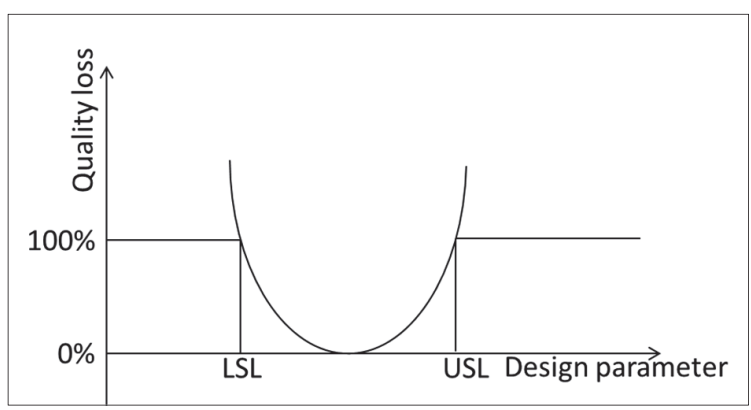

Fig. 1. Quadratic quality loss function

Performance of a product between these specification limits is deemed to be acceptable. Taguchi did not accept this traditional definition of quality. He defined 'quality' as the deviation from on-target performance. According to him, "quality of a manufactured product is total loss generated by that product to 
society from the time it is shipped, other than any losses caused by intrinsic functions". By the term 'loss' Taguchi referred to the loss caused by the variability of the function and loss caused by harmful side effects. It thus signifies that products with acceptable quality incur the lowest quality loss, particularly zero. When a product moves from its target value, it will cause loss even if the product lies or not within the set specification limits. On the other hand, the engineering experience shows that quality degrades, with some exceptions, continuously. Taguchi proposed that this performance degradation can be measured as a deviation from some target value as a quadratic quality loss function, as shown in Fig. 1 and asserted that the degradation can be related to a loss in value to the consumer.

Thus, the objective of quality loss function as a quality improvement tool is to minimize the total losses to society. Subsequently, the quadratic quality loss function approach has been applied in on-line and off-line quality control. The advantage of quadratic loss function mainly lies on its simplicity and comprehensiveness. In order to reduce the expected losses with respect to quadratic loss function, the process mean should be close to the target and process standard deviation should be small. Thus, if a quality characteristic concentrates on target value with minimum standard deviation, it can be said that the product has minimum quality loss. Quality loss function approach has already become a popular tool for evaluating the quality level of products, mainly in one-dimensional case (Târcolea \& Paris, 2011). The concept of quality loss function thus forms the basis for a quality evaluation system and an appropriately chosen loss function should be competent in evaluating the quality of a given product. The idea of multivariate loss function was proposed by Artiles-León (1996-97), and Ma and Zhao (2004) then modified it to make it more suitable for real time applications. It has been observed that most of the quality characteristics are of 'nominal-the-best' type ( $N$-type), where each product has an ideal value for each of its characteristics from the viewpoint of the customers/end users. This ideal value is often referred to as the target value and the corresponding loss is always proportional to the square of the deviation of the said quality characteristic from its target value. Taguchi proposed the following quadratic loss function to estimate the quality losses (Taguchi et al., 2004).

$$
\operatorname{Loss}(y)=k(y-t)^{2}
$$

where $y$ is the quality characteristic of a product, $t$ is the target value for $x$, the quality characteristic and $k$ is a quality loss coefficient. It is required to determine the value of $k$ so that the above-mentioned equation can be able to appropriately approximate the actual economical loss within the interest domain. In order to estimate the value of $k$ so that the loss function remains insensitive to the system of units employed to measure the corresponding quality characteristic, Artiles-León (1996-97) assumed that the relationship between each quality characteristic and design variable could be predicted, the target value would belong to the centre of the specifications (symmetric loss function), and the loss function would become unity whenever the quality characteristic value would be at its upper or lower specification limit. Thus, the value of $k$ can be expressed as follows:

$$
k=\left(\frac{2}{U S L-L S L}\right)^{2} \text {. }
$$

Substituting the value of $k$ in Eq. (1), the loss function becomes:

$$
L(y)=4\left(\frac{y(x)-t}{U S L-L S L}\right)^{2},
$$

where $U S L$ and $L S L$ are the upper and lower specification limits of the considered quality characteristic respectively. As this 'standardized' loss function is dimensionless, loss functions corresponding to several quality characteristics for a product can be added when the quality characteristics are uncorrelated, and Artiles-León (1996-97) defined the total standardized loss function (TSLoss) corresponding to quality characteristics, $Y_{1}, Y_{2}, \ldots, Y_{n}$, as below:

$$
\operatorname{TSLoss}\left(Y_{1}, Y_{2}, \ldots, Y_{n}, X, T\right)=4 \sum_{i=1}^{n}\left(\frac{Y_{i}(X)-t_{i}}{U S L_{i}-L S L_{i}}\right)^{2},
$$


where $t_{i}, L S L_{i}$ and $U S L_{i}(i=1,2, \ldots, n)$ are the target value, lower specification limit and upper specification limit respectively, for $i^{\text {th }}$ quality characteristic $Y_{i}$, in which the quality characteristic $Y_{i}$ is a function of design variables $X^{\prime} s$. The loss function in Eq. (3) can be treated as a practical tool to multi-response problems and can be easily implemented. However, it has sudden drawbacks which need to be overcome. Firstly, it is not suitable for 'smaller-the-better' type ( $S$-type) and 'larger-the- better' type ( $L$-type) of quality characteristics. On the other hand, it does not consider the correlation structure between different responses. Thus, it becomes necessary to modify it to make it more suitable for real time applications. Ma and Zhao (2004) developed an improved multivariate loss function approach for different quality characteristics, i.e. 'larger-the-better' ( $L$-type), 'smaller-the-better' $(S$-type) and 'nominal-the- best' $(N$ type) (Târcolea \& Paris, 2011). For an $L$-type quality characteristic $\left(Y_{i}\right)$, let $Y_{L_{i}}$ be the minimum acceptable value and $Y_{U_{i}}$ be the maximum value beyond which there is no further improvement in the product performance. Now, the dimensionless loss function for $L$-type quality characteristic can be expressed as follows:

$$
L\left(Y_{i}(X), X, Y_{U_{i}}\right)=\left(\frac{Y_{i}(X)-Y_{U_{i}}}{Y_{U_{i}}-Y_{L_{i}}}\right)^{2} .
$$

Similarly, for an $S$-type quality characteristic, the loss function can be modelled as below:

$$
L\left(Y_{i}(X), X, Y_{L_{i}}\right)=\left(\frac{Y_{i}(X)-Y_{L_{i}}}{Y_{U_{i}}-Y_{L_{i}}}\right)^{2} .
$$

Thus, as an extension of Artiles-León's loss function, all the three types of loss function now become dimensionless and can be added to form a more generalized multivariate loss function, as given below:

$$
L(Y(X), X)=\sum_{i \in N} 4\left(\frac{Y_{i}(X)-t_{i}}{U S L_{i}-L S L_{i}}\right)^{2}+\sum_{j \in L}\left(\frac{Y_{j}(X)-Y_{U_{j}}}{Y_{U_{j}}-Y_{L_{j}}}\right)^{2}+\sum_{l \in S}\left(\frac{Y_{l}(X)-Y_{L_{l}}}{Y_{U_{l}}-Y_{L_{l}}}\right)^{2},
$$

where $N, L$ and $S$ are the numbers of $N$-type, $L$-type and $S$-type quality characteristics.

\section{Illustrative examples}

In this paper, the multivariate quality loss function approach is applied for multi-response optimization of NTM processes and its superiority over the other state-of-the-art optimization methods is well validated with the help of three illustrative examples.

\subsection{Example 1}

Mehrvar et al. (2017) considered four ECM process parameters, i.e. applied voltage, tool feed rate, electrolyte flow rate and electrolyte concentration to investigate their effects on MRR and SR. Each of those process parameters was set at five different levels, as shown in Table 1, and based on the central composite second order rotatable design plan, 31 experiments were conducted while taking stainless steel as the work material. The results of those 31 experiments are detailed out in Table 2 . The machining time was fixed for $2 \mathrm{~min}$ and the inter-electrode gap was set at $0.6 \mathrm{~mm}$.

\section{Table 1}

ECM process parameters along with their levels (Mehrvar et al., 2017)

\begin{tabular}{lccccccc}
\hline \multirow{2}{*}{ Process parameter } & \multirow{2}{*}{ Symbol } & Unit & \multicolumn{5}{c}{ Level } \\
\cline { 4 - 8 } & & & -2 & -1 & 0 & 1 & 2 \\
\hline Applied voltage & $x_{1}$ & $\mathrm{~V}$ & 10 & 15 & 20 & 25 & 30 \\
Tool feed rate & $x_{2}$ & $\mathrm{~mm} / \mathrm{min}$ & 0.2 & 0.3 & 0.4 & 0.5 & 0.6 \\
Electrolyte flow rate & $x_{3}$ & $1 / \mathrm{min}$ & 5 & 6 & 7 & 8 & 9 \\
Electrolyte concentration & $x_{4}$ & $\mathrm{~g} / \mathrm{l}$ & 50 & 100 & 150 & 200 & 250 \\
\hline
\end{tabular}


Table 2

Experimental results for ECM process (Mehrvar et al., 2017)

\begin{tabular}{|c|c|c|c|c|c|c|}
\hline \multirow{2}{*}{ Exp. No. } & \multicolumn{4}{|c|}{ Process parameter } & \multicolumn{2}{|c|}{ Response } \\
\hline & $x_{1}$ & $x_{2}$ & $x_{3}$ & $x_{4}$ & $\operatorname{MRR}(\mathrm{g} / \mathrm{min})$ & $\mathrm{SR}(\mu \mathrm{m})$ \\
\hline 1 & -1 & -1 & -1 & -1 & 0.1253 & 0.76 \\
\hline 2 & 1 & -1 & -1 & -1 & 0.2134 & 1.08 \\
\hline 3 & -1 & 1 & -1 & -1 & 0.1547 & 0.89 \\
\hline 4 & 1 & 1 & -1 & -1 & 0.2361 & 1.13 \\
\hline 5 & -1 & -1 & 1 & -1 & 0.1246 & 0.84 \\
\hline 6 & 1 & -1 & 1 & -1 & 0.2107 & 1.16 \\
\hline 7 & -1 & 1 & 1 & -1 & 0.1569 & 0.96 \\
\hline 8 & 1 & 1 & 1 & -1 & 0.2525 & 1.31 \\
\hline 9 & -1 & -1 & -1 & 1 & 0.1673 & 1.29 \\
\hline 10 & 1 & -1 & -1 & 1 & 0.2921 & 1.94 \\
\hline 11 & -1 & 1 & -1 & 1 & 0.1975 & 1.63 \\
\hline 12 & 1 & 1 & -1 & 1 & 0.3218 & 2.21 \\
\hline 13 & -1 & -1 & 1 & 1 & 0.1779 & 1.47 \\
\hline 14 & 1 & -1 & 1 & 1 & 0.2979 & 2.15 \\
\hline 15 & -1 & 1 & 1 & 1 & 0.2019 & 1.78 \\
\hline 16 & 1 & 1 & 1 & 1 & 0.3235 & 2.49 \\
\hline 17 & -2 & 0 & 0 & 0 & 0.1154 & 1.22 \\
\hline 18 & 2 & 0 & 0 & 0 & 0.3379 & 2.17 \\
\hline 19 & 0 & -2 & 0 & 0 & 0.1989 & 1.12 \\
\hline 20 & 0 & 2 & 0 & 0 & 0.2755 & 1.51 \\
\hline 21 & 0 & 0 & -2 & 0 & 0.1927 & 1.12 \\
\hline 22 & 0 & 0 & 2 & 0 & 0.2194 & 1.35 \\
\hline 23 & 0 & 0 & 0 & -2 & 0.1365 & 0.72 \\
\hline 24 & 0 & 0 & 0 & 2 & 0.2696 & 2.45 \\
\hline 25 & 0 & 0 & 0 & 0 & 0.2351 & 1.00 \\
\hline 26 & 0 & 0 & 0 & 0 & 0.2291 & 0.98 \\
\hline 27 & 0 & 0 & 0 & 0 & 0.2250 & 1.02 \\
\hline 28 & 0 & 0 & 0 & 0 & 0.2238 & 0.96 \\
\hline 29 & 0 & 0 & 0 & 0 & 0.2220 & 1.04 \\
\hline 30 & 0 & 0 & 0 & 0 & 0.2275 & 0.95 \\
\hline 31 & 0 & 0 & 0 & 0 & 0.2232 & 1.02 \\
\hline
\end{tabular}

Now, based on the experimental data, Mehrvar et al. (2017) developed the following two response surface methodology (RSM)-based equations to show the main, second order and interaction effects of the four ECM process parameters on MRR and SR.

$$
\begin{aligned}
Y(M R R) & =-0.3374+0.0055 x_{1}-0.0496 x_{2}+0.0755 x_{3}+0.00072 x_{4}-0.00001449 x_{1}^{2}+0.2275 x_{2}^{2} \\
& -0.00551 x_{3}^{2}-0.00000250 x_{4}^{2}+0.00049 x_{1} x_{2}+0.00005875 x_{1} x_{3}+0.000034875 x_{1} x_{3} \\
& +0.0072 x_{2} x_{3}-0.00020875 x_{2} x_{4}+0.000009125 x_{3} x_{4} \\
Y(S R)= & 8.75714-0.29921 x_{1}-7.01905 x_{2}-0.01042 x_{3}-0.0234 x_{4}+0.0069 x_{1}^{2}+7.74256 x_{2}^{2} \\
& +0.05743 x_{3}^{2}+0.00005794 x_{4}^{2}-0.01125 x_{1} x_{2}+0.00338 x_{1} x_{3}+0.00035 x_{1} x_{3}+0.08125 x_{2} x_{3} \\
& +0.01013 x_{2} x_{4}+0.00051 x_{3} x_{4}
\end{aligned}
$$

Later, differential evolutionary algorithm was employed for solving the related single and multi-objective optimization problems while developing the optimal Pareto front. It was claimed that the adopted algorithm would be able to determine a set of ECM process parameters for having global optimal values of the responses with reasonable computational cost and time. While applying differential evolutionary algorithm for simultaneous optimization of the two responses, Mehrvar et al. (2017) derived the optimal combination of the process parameters as applied voltage $=24.78 \mathrm{~V}$, tool feed rate $=0.49 \mathrm{~mm} / \mathrm{min}$, electrolyte flow rate $=6.84 \mathrm{l} / \mathrm{min}$ and electrolyte concentration $=134.15 \mathrm{~g} / \mathrm{l}$ with a MRR value of 0.28 $\mathrm{g} / \mathrm{min}$ and SR of $1.36 \mu \mathrm{m}$. Now, when the multivariate quality loss function approach is adopted for parametric optimization of the considered ECM process, the acceptable range for MRR is decided to lie 
between $0.25 \mathrm{~g} / \mathrm{min}$ and $0.50 \mathrm{~g} / \mathrm{min}$. On the other hand, the acceptable range for SR is from $0.5 \mu \mathrm{m}$ to $1.5 \mu \mathrm{m}$. These ranges are decided based on the observed MRR and SR values in Table 2. As MRR is an $L$-type quality characteristic, its higher value is always desired. Similarly, SR being an $S$-type quality characteristic, it is always preferable to have its lower value. Based on these considerations, the USL and LSL values for the two responses are set in the objective function of the developed multi-response optimization problem. Of course, the optimal settings should be constrained to reside on or within the sphere defined by the experimental design plan. Thus, according to the loss function provided in Eq. (7), the multi-response optimization problem can be expressed as follows:

$$
\operatorname{Min} L(Y, X)=\left(\frac{y(M R R)-0.5}{0.25}\right)^{2}+\left(\frac{y(S R)-0.5}{1}\right)^{2}
$$

subject to

$0.25 \leq y(M R R) \leq 0.50$

$0.50 \leq y(S R) \leq 1.50$

$x_{1}^{2}+x_{2}^{2}+x_{3}^{2}+x_{4}^{2} \leq 2^{2}$

The above-mentioned non-linear optimization problem is now solved using LINGO software and the derived results are shown in Table 3. It is observed that a maximum value of MRR and a minimum value of SR are simultaneously achieved at the coded values of $x_{1}, x_{2}, x_{3}$ and $x_{4}$ as $0.2480,-1.6213,0.9892$ and 0.1942 respectively. After de-coding, the optimal parametric mix for the considered ECM process is thus attained at applied voltage $=21.24 \mathrm{~V}$, tool feed rate $=0.2379 \mathrm{~mm} / \mathrm{min}$, electrolyte flow rate $=7.9892$ $1 / \mathrm{min}$ and electrolyte concentration $=159.71 \mathrm{~g} / 1$ with a MRR of $0.40 \mathrm{~g} / \mathrm{min}$ and SR of $1 \mu \mathrm{m}$. It is interestingly noticed that with this optimal parametric mix, the MRR value is increased by $30 \%$ and SR value is trimmed down by $26.47 \%$ as compared to those obtained by Mehrvar et al. (2017). From the singleobjective optimization results of Mehrvar et al. (2017), it is observed that maximum MRR would occur at higher applied voltage, tool feed rate and electrolyte concentration, while moderate to low level of applied voltage and electrolyte concentration would be responsible for minimum SR. In order to have a trade-off between these two conflicting quality characteristics (responses), it is thus recommended to operate the considered ECM process at higher values of applied voltage, electrolyte flow rate and electrolyte concentration, and lower value of tool feed rate. Table 3 also exhibits a comparison of the derived optimal solutions with those achieved while employing the other popular multi-objective optimization approaches, i.e. desirability function method (Derringer and Suich, 1980), distance function method (Khuri. and Conlon, 1981; Jing and Yongfan, 2017) and mean squared error method (Vining, 1998). In all the cases, the achieved MRR and SR values are observed to be worst as compared to those derived using the multivariate quality loss function approach.

Table 3

Comparison of the optimal parametric settings for ECM process

\begin{tabular}{ccccccc}
\hline Optimization approach & \multicolumn{3}{c}{ Variable } & \multicolumn{3}{c}{ Response } \\
\cline { 2 - 7 } & $x_{1}$ & $x_{2}$ & $x_{3}$ & $x_{4}$ & MRR & SR \\
\hline Multivariate loss function approach & 0.2480 & -1.6213 & 0.9892 & 0.1942 & 0.4 & 1 \\
Differential evolutionary algorithm & 0.9560 & 0.9 & -0.16 & -0.317 & 0.28 & 1.36 \\
Desirability function method & 2 & 2 & 2 & -2 & 0.2589 & 1.2 \\
Distance function method & 0.7923 & -1.5851 & 0.9091 & -1.1820 & 0.3700 & 1.2 \\
Mean squared error method & 0.4002 & -1.5522 & 1.0255 & 0.3548 & 0.3670 & 1.151 \\
\hline
\end{tabular}

\subsection{Example 2}

While performing EDM operation on AISI 316LN stainless steel using copper as an electrode, Majumder et al. (2014) predicted the optimal settings of three process parameters, i.e. supply current, pulse-on time and pulse-off time for achieving maximum MRR and minimum volumetric electrode wear ratio (EWR). Each of those process parameters was set at three different levels, as provided in Table 4, and based on Box-Behnken response surface design plan, 15 experiments were carried out at different combinations 
of the considered process parameters. The detailed experimental results are exhibited in Table 5. Majumder et al. (2014) then employed desirability-based multi-objective particle swarm optimization (PSO) (original), desirability-based multi-objective PSO (inertia weight) and desirability-based multi-objective PSO (constriction factor) methods for multi-objective optimization of the responses. It was concluded that the desirability-based multi-objective PSO (constriction factor) method outperformed the other two approaches with respect to the achieved values of both the responses.

Table 4

EDM process parameters and their levels (Majumder et al., 2014)

\begin{tabular}{ccccccc}
\hline Process parameter & \multirow{2}{*}{ Symbol } & \multirow{2}{*}{ Unit } & \multicolumn{3}{c}{ Level } \\
\cline { 5 - 7 } & & & -1 & 0 & 1 \\
\hline Supply current (A) & $x_{1}$ & $\mathrm{~A}$ & 3 & 7 & 11 \\
Pulse-on duration (ON) & $x_{2}$ & $\mu \mathrm{s}$ & 8000 & 10000 & 12000 \\
Pulse-off duration (OFF) & $x_{3}$ & $\mu \mathrm{s}$ & 8000 & 10000 & 12000 \\
\hline
\end{tabular}

\section{Table 5}

Experimental plan and results for EDM process (Majumder et al., 2014)

\begin{tabular}{|c|c|c|c|c|c|}
\hline \multirow{2}{*}{ Exp. No. } & \multicolumn{3}{|c|}{ Process parameter } & \multicolumn{2}{|c|}{ Response } \\
\hline & $\mathrm{A}$ & ON & OFF & $\operatorname{MRR}\left(\mathrm{mm}^{3} / \mathrm{sec}\right)$ & Volumetric EWR (\%) \\
\hline 1 & 0 & 0 & 0 & 8.13 & 5.994 \\
\hline 2 & 0 & 1 & -1 & 2.3173 & 4.492 \\
\hline 3 & 0 & 0 & 0 & 8.438 & 6.01 \\
\hline 4 & -1 & -1 & 0 & 3.429 & 4.7926 \\
\hline 5 & 1 & 0 & -1 & 4.008 & 14.487 \\
\hline 6 & -1 & 0 & 1 & 3.625 & 5.496 \\
\hline 7 & 0 & -1 & -1 & 5.003 & 4.13 \\
\hline 8 & 1 & 1 & 0 & 4.919 & 6.331 \\
\hline 9 & 0 & 1 & 1 & 5.82 & 4.5283 \\
\hline 10 & -1 & 1 & -1 & 3.03 & 4.8789 \\
\hline 11 & 0 & 0 & 0 & 8.42 & 5.98 \\
\hline 12 & -1 & 1 & 0 & 3.27 & 4.055 \\
\hline 13 & 1 & -1 & 0 & 4.743 & 16.32 \\
\hline 14 & 1 & 0 & 1 & 10.011 & 4.1759 \\
\hline 15 & 0 & -1 & 1 & 4.2307 & 6.727 \\
\hline
\end{tabular}

Based on the experimental data, the following two RSM-based equations were developed interrelating the responses with the three EDM process parameters which are later employed for the multivariate quality loss function-based approach.

$$
\begin{aligned}
Y(M R R) & =8.43060+1.54088 x_{1}+0.11508 x_{2}+1.16605 x_{3}-2.75730 x_{1}^{2}-3.58305 x_{2}^{2} \\
- & 2.00480 x_{3}^{2}+0.08375 x_{1} x_{2}+1.852 x_{1} x_{3}+0.56875 x_{2} x_{3} \\
Y(E W R) & =5.9940+2.7614 x_{1}-1.5704 x_{2}-0.8826 x_{3}+2.0854 x_{1}^{2}-0.2047 x_{2}^{2} \\
- & 0.8199 x_{3}^{2}-2.31228 x_{1} x_{2}-2.7321 x_{1} x_{3}-0.6402 x_{2} x_{3}
\end{aligned}
$$

Now, based on the above-mentioned RSM-based equations and experimental data of Table 5, the following multi-response optimization problem in the form of multivariate loss function is formulated. It is worthwhile to mention here that MRR is an $L$-type and EWR is an $S$-type quality characteristics.

$$
\begin{aligned}
& \min L(Y, X)=\left(\frac{y(M R R)-10}{2}\right)^{2}+\left(\frac{y(E W R)-3}{1}\right)^{2} \\
& \text { subject to } \\
& 8 \leq y(M R R) \leq 10 \\
& 3 \leq y(E W R) \leq 4 \\
& x_{1}^{2}+x_{2}^{2}+x_{3}^{2} \leq 1^{2}
\end{aligned}
$$


While solving this multi-response optimization problem using LINGO software, it is observed that a maximum MRR value of $9.0776 \mathrm{~mm}^{3} / \mathrm{sec}$ and a minimum EWR value of $3.5 \%$ are attained at the coded parametric settings of the considered EDM process as $x_{1}=0.4509, x_{2}=0.0616$ and $x_{3}=0.5078$. On the other hand, while applying the desirability-based multi-objective PSO (constriction factor) approach, Majumder et al. (2014) achieved a MRR value of $8.4306 \mathrm{~mm}^{3} / \mathrm{sec}$ and an EWR value of $3.95 \%$ at the optimal settings of $x_{1}=0, x_{2}=0$ and $x_{3}=1$. It is noticed that in the multivariate loss function approach, there is an increase in MRR by $7.67 \%$ and EWR is decreased by $11.39 \%$. In this approach, the actual parametric mix for the considered EDM process is observed as supply current $=8.8036 \mathrm{~A}$, pulse-on duration $=10123.2 \mu$ s and pulse-off duration $=11015.6 \mu$ s. Thus, in this EDM operation, for having the maximum MRR and minimum EWR values, it is always recommended to approximately set higher values for all the considered process parameters. Table 6 compares the optimization performance of multivariate loss function approach with that of the other methods, like desirability function, distance function and mean squared method, and it is revealed that this adopted method supersedes others with respect to the achieved values of both MRR and EWR.

\section{Table 6}

Comparison of the optimal parametric settings for EDM process

\begin{tabular}{|c|c|c|c|c|c|}
\hline \multirow{2}{*}{ Optimization approach } & \multicolumn{3}{|c|}{ Variable } & \multicolumn{2}{|c|}{ Response } \\
\hline & $x_{1}$ & $x_{2}$ & $x_{3}$ & MRR & EWR \\
\hline Multivariate loss function approach & 0.4509 & 0.0616 & 0.5078 & 9.0776 & 3.5 \\
\hline Desirability-based multi-objective PSO & 0 & 0 & 1 & 8.4306 & 3.95 \\
\hline Desirability function method & 0 & 1 & 1 & 6.5281 & 5.344 \\
\hline Distance function method & 0.0382 & 0.0029 & 0.0290 & 8.52 & 3.60 \\
\hline Mean squared error method & 0.3426 & 0.0339 & 0.3151 & 9.01 & 3.61 \\
\hline
\end{tabular}

\subsection{Example 3}

In this example, the experimental data of Sivaprakasam et al. (2013) are considered for multi-objective optimization of the responses using the adopted approach. Based on a central composite design plan, Sivaprakasam et al. (2013) conducted 20 experiments in a micro-WEDM set-up while taking into account three process parameters, i.e. voltage, capacitance and feed rate, and three responses, i.e. MRR (in $\mathrm{mm}^{3} / \mathrm{min}$ ), kerf width (KW) (in $\mu \mathrm{m}$ ) and SR (in $\mu \mathrm{m}$ ). Each of those process parameters had three different levels, as shown in Table 7. The derived experimental data are exhibited in Table 8. Using desirability function approach, Sivaprakasam et al. (2013) determined the optimal values of MMR, KW and SR responses as $0.0260 \mathrm{~mm}^{3} / \mathrm{min}, 87 \mu \mathrm{m}$ and $0.97 \mu \mathrm{m}$ respectively at a parametric combination of voltage $=80 \mathrm{~V}$, capacitance $=0.01 \mu \mathrm{F}$ and feed rate $=15 \mu \mathrm{m} / \mathrm{sec}$. In this example, MRR is an $L$-type response, whereas, KW and SR are $S$-type responses.

Table 7

Micro-WEDM process parameters and their levels (Sivaprakasam et al., 2013)

\begin{tabular}{|c|c|c|c|c|c|}
\hline \multirow{2}{*}{ Process parameter } & \multirow{2}{*}{ Symbol } & \multirow{2}{*}{ Unit } & \multicolumn{3}{|c|}{ Level } \\
\hline & & & -1 & 0 & 1 \\
\hline Voltage & $x_{1}$ & $\mathrm{~V}$ & 80 & 90 & 100 \\
\hline Capacitance & $x_{2}$ & $\mu \mathrm{F}$ & 0.01 & 0.1 & 0.4 \\
\hline Feed rate & $x_{3}$ & $\mu \mathrm{m} / \mathrm{sec}$ & 5 & 10 & 15 \\
\hline
\end{tabular}

Based on the RSM technique, three regression models were developed highlighting the influences of the micro-WEDM process parameters on the considered responses. Using these three regression equations and employing the multivariate loss function approach, the corresponding optimization problem is now formulated which is subsequently solved using LINGO software. It is interestingly noticed that in this approach, the value of MRR is increased to $0.0893 \mathrm{~mm}^{3} / \mathrm{min}$, and the values of KW and SR are decreased to $85.73 \mu \mathrm{m}$ and $0.95 \mu \mathrm{m}$ respectively. From the experimental investigations of Sivaprakasam et al. (2013), it is observed that an increase in voltage causes higher MRR value. Similarly, MRR also tends to increase with increase in capacitance. Increase in MRR is usually responsible for higher SR. Higher 
feed rate also causes attainment of higher MRR. On the other hand, minimum KW can be achieved at lower value of voltage.

Table 8

Experimental data for micro-WEDM process (Sivaprakasam et al., 2013)

\begin{tabular}{|c|c|c|c|c|c|c|}
\hline \multirow{2}{*}{ Exp. No. } & \multicolumn{3}{|c|}{ Variables } & \multicolumn{3}{|c|}{ Response } \\
\hline & $x_{1}$ & $x_{2}$ & $x_{3}$ & $\operatorname{MRR}\left(\mathrm{mm}^{3} / \mathrm{min}\right)$ & $\mathrm{KW}(\mu \mathrm{m})$ & $\mathrm{SR}(\mu \mathrm{m})$ \\
\hline 1 & 0 & 0 & 0 & 0.025084 & 97 & 2.19 \\
\hline 2 & 0 & 0 & 0 & 0.025694 & 97 & 1.77 \\
\hline 3 & 1.68 & 0 & 0 & 0.027545 & 96 & 2.07 \\
\hline 4 & 0 & 1.68 & 0 & 0.025992 & 94 & 3.2 \\
\hline 5 & 1 & 1 & -1 & 0.020042 & 96 & 3.06 \\
\hline 6 & -1 & -1 & -1 & 0.015232 & 87 & 1.1 \\
\hline 7 & 0 & -1.68 & 0 & 0.024028 & 88 & 0.92 \\
\hline 8 & 0 & 0 & 0 & 0.026618 & 97 & 2.07 \\
\hline 9 & 0 & 0 & 0 & 0.027551 & 97 & 2.17 \\
\hline 10 & 0 & 0 & 0 & 0.026297 & 96 & 1.72 \\
\hline 11 & -1 & -1 & 1 & 0.025925 & 87 & 0.97 \\
\hline 12 & 1 & 1 & 1 & 0.033847 & 94 & 3.3 \\
\hline 13 & 0 & 0 & 1.68 & 0.035446 & 96 & 1.97 \\
\hline 14 & -1 & 1 & 1 & 0.035714 & 95 & 2.27 \\
\hline 15 & 1 & -1 & -1 & 0.021056 & 97 & 1.15 \\
\hline 16 & 1 & -1 & 1 & 0.03675 & 97 & 1.12 \\
\hline 17 & -1.68 & 0 & 0 & 0.022 & 90 & 1.16 \\
\hline 18 & 0 & 0 & 0 & 0.025463 & 96 & 2.15 \\
\hline 19 & 0 & 0 & -1.68 & 0.017504 & 97 & 1.97 \\
\hline 20 & -1 & 1 & -1 & 0.018968 & 98 & 2.55 \\
\hline
\end{tabular}

With the increment in voltage, the SR tends to increase noticeably. The SR response also increases when capacitance is increased. Keeping all these findings in mind, it is thus advised to operate the considered micro-WEDM process at lower values of voltage and capacitance, and higher value of feed rate so as to have maximum MRR, and minimum KW and SR values. A comparison between the derived optimal parametric settings (coded) for the considered micro-WEDM process and those attained by the other approaches are provided in Table 9. These observations also assure the superiority of multivariate quality loss function approach over the other considered techniques as an effective multi-objective optimization tool.

$$
\begin{aligned}
& \begin{aligned}
Y(M R R)= & 0.26194+0.017538 x_{1}+0.12824 x_{2}+0.060791 x_{3}-0.021428 x_{1} x_{2}
\end{aligned} \\
& \begin{aligned}
Y(K W)= & 96.69807+1.98367 x_{1}+1.83723 x_{2}-0.48926 x_{3}-2.87500 x_{1} x_{2}-0.62500 x_{2} x_{3} \\
& -1.18272 x_{1}^{2}-1.88983 x_{2}^{2}
\end{aligned} \\
& Y(S R)=2.03491+0.24533 x_{1}+0.78748 x_{2}+0.17750 x_{1} x_{2}-0.12728 x_{1}^{2} \\
& \min L(Y, X)=\left(\frac{y(M R R)-0.035}{0.01}\right)^{2}+\left(\frac{y(K W)-85}{5}\right)^{2}+\left(\frac{y(S R)-0.9}{0.1}\right)^{2}
\end{aligned}
$$

subject to

$$
\begin{aligned}
& 0.025 \leq y(M R R) \leq 0.035 \quad 85 \leq y(K W) \leq 90 \quad 0.9 \leq y(S R) \leq 1 \\
& x_{1}^{2}+x_{2}^{2}+x_{3}^{2} \leq 1.68^{2}
\end{aligned}
$$

Table 9

Comparison of the optimal parametric settings for micro-WEDM process

\begin{tabular}{ccccccc}
\hline Optimization approach & \multicolumn{3}{c}{ Variable } & \multicolumn{3}{c}{ Response } \\
\cline { 2 - 7 } & $x_{1}$ & $x_{2}$ & $x_{3}$ & MRR & KW & SR \\
\hline Multivariate loss function approach & -0.7933 & -0.8289 & -1.2512 & 0.0893 & 85.73 & 0.95 \\
Desirability function method & -1 & -1 & 1 & 0.0260 & 87 & 0.97 \\
Distance function method & -0.810 & -0.856 & -1.25 & 0.0876 & 86.31 & 0.9626 \\
Mean squared error method & -0.0014 & -0.012 & 0 & 0.035 & 86 & 0.96 \\
\hline
\end{tabular}




\section{Conclusions}

In non-traditional as well as conventional machining processes, it is always desired to explore their fullest machining potential while setting the controllable process parameters at their optimal operating levels. Several mathematical approaches already exist which can determine the optimal settings of the NTM process parameters separately for each of the responses. As there is always more than one response in NTM processes, it is almost impossible/impractical to set different parametric settings for different responses all at a time in a particular machining set-up. Thus, in this paper, a multivariate quality loss function approach is adopted to identify a single parametric setting which would almost simultaneously optimize all the responses of a considered NTM process. The adopted approach would guide the concerned process engineers in identifying the desired combinations of various parameters for different NTM processes. The derived results are also compared with those obtained by the other popular methods which prove the superiority of the adopted approach over them as an efficient multi-objective optimization tool. This approach can also be employed to other NTM and conventional material removal processes to determine the optimal parametric mixes so as to achieve the desired responses under constrained machining environments.

\section{References}

Antony, J. (2001). Simultaneous optimisation of multiple quality characteristics in manufacturing processes using Taguchi's quality loss function. International Journal of Advanced Manufacturing Technology, 17(2), 134-138.

Artiles-León, N. (1996-97). A pragmatic approach to multi-response problems using loss functions. Quality Engineering, 9(2), 213-220.

Bhuyan, B.K., \& Yadava,V. (2014). Modelling and optimisation of travelling wire electro-chemical spark machining process. International Journal of Industrial and Systems Engineering, 18(2), 139-158.

Das Mohapatra, K., \& Sahoo, S. K. (2014). Parametric optimization of wire EDM process for gear cutting. Journal of Basic and Applied Engineering Research, 1(3), 78-84.

Datta, S., \& Mahapatra, S.S. (2010). Simultaneous optimization of correlated multiple surface quality characteristics of mild steel turned product. Intelligent Information Management, 2(1), 26-39.

Dave, H.K., Desai, K.P., \& Raval, H.K. (2012). Optimisation of multiple response characteristics in orbital electro discharge machining of Inconel 718 using Taguchi's loss function. International Journal of Manufacturing Technology and Management, 25(1-3), 78-94.

Derringer, G., \& Suich, R. (1980). Simultaneous optimization of several response variables. Journal of Quality Technology, 12(4), 214-219.

Dhobe, S. D., Doloi, B., \& Bhattacharyya, B. (2014). Optimisation of ECM process during machining of titanium using quality loss function. International Journal of Manufacturing Technology and Management, 28(1-3), 19-38.

Gaitonde, V.N., \& Karnik, S.R. (2012). Selection of optimal process parameters for minimizing burr size in drilling using Taguchi's quality loss function approach. Journal of the Brazilian Society of Mechanical Science and Engineering, $X X X I V(3), 238-245$.

Hansda, S., \& Banerjee, S. (2014). Multi characteristics optimization using Taguchi quality loss function with varying weightage of responses in drilling of GFRP composite. In: Proceedings of 5th International \& 26th All India Manufacturing Technology, Design and Research Conference, India, 1-6.

Jing, W., \& Yongfan, L. (2017). A robustness generalized distance function approach for multiresponse robust optimization. Procedia Engineering, 174, 748-755.

Khuri, A.I., \& Conlon, M. (1981).Simultaneous optimization of multiple responses represented by polynomial regression functions. Technometrics, 23(4), 363-375.

Koranne, J., Verma, M., \& Khandelwal, A.K. (2014). Multi-objective optimization of turning process by using quality loss function. Journal of Harmonized Research in Engineering, 2(2), 259-265.

Kumar, V., \& Kumari, N. (2012). Modeling and multiple performance optimization ofultrasonic micro-hole machining of PCD using fuzzy logic and Taguchi quality loss function.Advances in Materials Research, $1(2), 129-146$. 
Kumar, J. (2014). Investigations into the surface quality and micro-hardness in the ultrasonic machining of titanium (ASTM GRADE-1). Journal of the Brazilian Society of Mechanical Sciences and Engineering, 36(4), 807-823.

Ma, Y., \& Zhao, F. (2004). An improved multivariate loss function approach to optimization. Journal of Systems Science and Systems Engineering, 13(3), 318-325.

Majumder, A., Das, P.K., Majumder, A., \& Debnath, M. (2014). An approach to optimize the EDM process parameters using desirability-based multi-objective PSO. Production \& Manufacturing Research, 2(1), 228-240.

Mehrvar, A., Basti, A., Jamali, A. (2017). Optimization of electrochemical machining process parameters: Combining response surface methodology and differential evolution algorithm. Proceeding of the Institution of Mechanical Engineers, Part E: Journal of Process Mechanical Engineering, 231(6), 1114-1126.

Padhy, J. P., Dewangan, S., \& Biswas, C. K. (2014).Optimization of multi-objective optimization of machining parameters of AJM using quality loss function. International Journal of Current Engineering and Technology, 3(2), 255-257.

Pandey, P.C., \& Shan, H.S. (1980). Modern Machining Processes. Tata McGraw-Hill Publishing Com. Ltd., New Delhi, India.

Periyanan, P.R., Natarajan, U., \& Yang, S.H. (2012).Multi-objective optimisation for the micro-end milling process using Taguchi quality loss function. International Journal of Productivity and Quality Management, 10(4), 484-497.

Periyanan, P.R., \& Natarajan, U. (2014).Optimization of multiple-quality characteristics in micro-WEDG process using Taguchi technique. International Journal of Quality \& Reliability Management, 31(2), 205-219.

Rahul, Datta, S., Biswal, B.B., \& Mahapatra, S.S. (2017). A novel satisfaction function and distance-based approach for machining performance optimization during electro-discharge machining on super alloy Inconel 718. Arabian Journal for Science and Engineering, 42(5), 1999-2020.

Sahoo, A.K., Mohanty, T. (2013). Optimization of multiple performance characteristics in turning using Taguchi's quality loss function: An experimental investigation. International Journal of Industrial Engineering Computations, 4(3), 325-336.

Singh, H., \& Kumar, P. (2006).Optimizing multi-machining characteristics through Taguchi's approach and utility concept. Journal of Manufacturing Technology Management, 17(2), 255-274.

Singh, G.K., Yadava, V., \& Kumar, R.(2012). Robust parameter design and multi-objective optimisation of electro-discharge diamond face grinding of HSS. International Journal of Machining and Machinability of Materials, 11(1), 1-19.

Sivaprakasam, P., Hariharan, P., \& Gowri, S. (2013). Optimization of micro-WEDM process of aluminum matrix composite (A413-B4C): A response surface approach. Materials and Manufacturing Processes, 28(12), 1340-1347.

Taguchi, G., Chowdhury, S., \& Wu, Y. (2004). Introduction to the Quality Loss Function. In Taguchi's Quality Engineering Handbook, John Wiley \& Sons, Inc., NJ, USA.

Târcolea, C., \& Paris, A.S. (2011). Loss functions used in the quality theory. University Politehnica of Bucharest Scientific Bulletin Series A, 73(1), 45-54.

Vining, G.G. (1998). A compromise approach to multiresponse optimization. Journal of Quality Technology, 30(4), 309-313.

Walia, R.S., Shan, H.S., \& Kumar, P. (2006). Multi-response optimization of CFAAFM process through Taguchi method and utility concept. Materials and Manufacturing Processes, 21(8), 907-914.

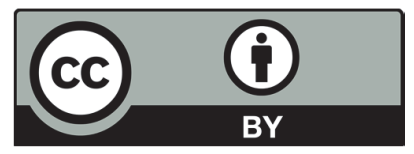

(C) 2018 by the authors; licensee Growing Science, Canada. This is an open access article distributed under the terms and conditions of the Creative Commons Attribution (CCBY) license (http://creativecommons.org/licenses/by/4.0/). 